\title{
Características físicas y perfil de diámetro de fibra de alpacas Huacaya del Centro Experimental La Raya (Puno, Perú), según edad y sexo
}

\author{
Physical characteristics and fibre diameter profile of Huacaya alpacas from La \\ Raya Experimental Centre (Puno, Peru), according to age and sex
}

\author{
Jesús E. Quispe Coaquiraa ${ }^{1,2}$, Edgar Apaza Zúñiga ${ }^{1}$, C. Uberto Olarte Daza ${ }^{1,2}$
}

\section{Resumen}

Se determinaron las características tecnológicas y el perfil del diámetro de fibra según edad (1, 3, 5 y 7 años) y sexo (macho y hembra) de alpacas Huacaya del Centro Experimental La Raya (Puno, Perú). Se tomaron muestras de vellón del costillar medio al momento de la esquila y se analizaron con el equipo OFDA 2000. Los datos fueron ajustados a regresión múltiple cuadrática para el análisis de las tasas de cambio. El diámetro promedio, factor de confort, índice de curvatura y longitud de fibra fueron estadísticamente similares entre sexos, mientras que para la edad, el diámetro incrementó (de $19.48 \pm 0.25$ a $24.82 \pm 0.80 \mu \mathrm{m}$ ), y disminuyó el factor de confort (de 98.15 a $86.95 \%$ ) y la longitud de fibra (de $102.18 \pm 12.92$ a $75.00 \pm 11.34 \mathrm{~mm}$ ), mientras que el índice de curvatura no mostró tendencia definida. Los parámetros que caracterizan el perfil del diámetro de fibra mostraron un solo valor máximo del diámetro medio de fibra, que mostró una curva cóncava con dos segmentos ascendente y descendente. Las tasas de cambio fueron positivas y negativas, aunque sus magnitudes fueron pequeñas. Se concluye que las alpacas del estudio presentan características tecnológicas propias y el perfil del diámetro configura un patrón característico estacional coincidente con la estacionalidad pluvial del Altiplano.

Palabras clave: textil, fibra, Huacaya

\footnotetext{
${ }^{1}$ Instituto de Investigación y Promoción de Camélidos Sudamericanos (IIPC), Puno, Perú

${ }^{2}$ Universidad Nacional del Altiplano, Puno, Perú

${ }^{3}$ E-mail: jesusquispecoaquira@gmail.com
} 
The technological characteristics and the fibre diameter profile were determined according to age (1, 3, 5, 7 years) and sex (male, female) of Huacaya alpacas from the La Raya Experimental Centre (Puno, Peru). Fleece samples were taken from the middle rib at shearing and analysed with the OFDA 2000 equipment. The data were adjusted to multiple quadratic regression for the analysis of the exchange rates. The average diameter, comfort factor, curvature index and fibre length were statistically similar between sexes, while for age, the diameter increased (from $19.48 \pm 0.25$ to $24.82 \pm 0.80 \mu \mathrm{m}$ ), and both the comfort factor (from 98.15 to $86.95 \%$ ) and the fibre length (from $102.18 \pm 12.92$ to $75.00 \pm 11.34 \mathrm{~mm}$ ) decreased, while the curvature index shows no defined trend. The parameters that characterize the fibre diameter profile showed a single maximum value of the mean fibre diameter, which showed a concave curve with two ascending and descending segments. Exchange rates were positive and negative, although their magnitudes were small. It is concluded that the alpacas in the study present their own technological characteristics and the diameter profile configures a characteristic seasonal pattern coinciding with the rainfall seasonality of the Altiplano.

Key words: textile, fibre, Huacaya

\section{INTRODUCCIÓN}

La alpaca (Vicugna pacos) es la especie doméstica de los camélidos sudamericanos que habita encima de los $3500 \mathrm{~m}$ en los Andes peruanos. El Perú posee 3685516 alpacas (Vicugna pacos), de las cuales el $79 \%$ es de la raza Huacaya y su mayor concentración se encuentra en la región Puno (42\%) (IV CENAGRO, 2013; Quispe et al., 2016). Asimismo, al igual que otros camélidos sudamericanos, convierte con eficiencia la diversificada vegetación de la pradera altoandina en productos de alta calidad como la fibra (Bustinza, 2001; Quispe et al., 2016). En los altos Andes, la producción de pastos tiene grandes variaciones dentro del año y entre años debido a los cambios climáticos durante la época lluviosa (noviembre-abril) y la época seca (mayo-octubre) (Quispe et al., 2016).

En el mundo del vestir, la fibra de alpaca ha logrado posicionarse por su excelente calidad. El 80\% se comercializa como mate- ria prima o intermedia para la elaboración de productos finales o como tejidos (MINCETUR, 2018). Según la SUNAT (2016), el 49\% se exporta a Estados Unidos, seguido de Alemania (10\%), Japón (5\%), Francia (5\%) y Reino Unido (5\%). Entre las propiedades apropiadas de la fibra para confección de prendas de vestir abrigadoras resaltan la finura y suavidad; además de la resistencia, comodidad, protección térmica y rendimientos al lavado e hilado (Cardellino y Mueller, 2008).

El menor o mayor aprecio de las fibras guarda relación directa con el grado de finura, el cual finalmente se expresa en el grado de confort de la prenda (Villarroel, 1991; Antonini y Vinella, 1997; Bustinza, 2001). Para la industria textil, la calidad de la fibra de alpaca está determinada por el color, el diámetro y longitud de la fibra; siendo la uniformidad de estas dos últimas la más apreciada. McGregor (2006) precisa que el diámetro promedio de fibra es el principal determinante de la calidad y la longitud de la fibra determina la ruta del procesamiento textil 
(Wang et al., 2003). Más aún, en el país, ambas características se utilizan para la categorización y clasificación de la fibra de alpaca (NTP 231.302.2004 y NTP 231.301.2004).

El diámetro de la fibra, desde la punta hasta la base, expresa los cambios ambientales ocurridos durante el periodo de crecimiento desde la última esquila. Puede mantenerse constante o variar creando un patrón característico a lo largo del año denominado el perfil del diámetro de la fibra (PDF) (Sacchero y Mueller, 2007). Al respecto, no se dispone de información precisa para las alpacas del Altiplano peruano que contribuya a delinear una mejor gestión de la producción de alpacas.

Son escasos los estudios sobre el PDF en las alpacas del país. En la sierra central se ha caracterizado en función al periodo de crecimiento y número de esquila, reportándose el diámetro medio de fibra $(22.05 \pm 0.88 \mu \mathrm{m})$, medias de diámetro mínimo $(20.11 \pm 0.79 \mu \mathrm{m})$ y máximo $(25.15 \pm 0.93 \mu \mathrm{m})$ y tasas de cambio descendente $(-0.094 \pm 0.011 \mu \mathrm{m}) \mathrm{y}$ ascendente $(0.108 \pm 0.024 \mu \mathrm{m})$ (Poma y Ventura, 2009) que permiten concluir que dichos parámetros están estrechamente vinculados a las variaciones de los factores medioambientales. Es decir, con los cambios que ocurren en el balance de nutrientes a causa de la estacionalidad pluvial -del estiaje o periodo lluvioso-; los que influyen directa e indirectamente en el crecimiento de la fibra y en las variaciones del diámetro a lo largo de la mecha, especialmente en alpacas de primera esquila.

El presente trabajo tuvo como objetivo caracterizar el diámetro medio de fibra (DMF), del factor de confort (FC), índice curvatura (IC) y longitud de fibra (LF); así como el perfil de diámetro de fibra (PDF) de alpacas Huacaya, según edad y sexo, del CIP La Raya de la Universidad Nacional del Altiplano.

\section{Materiales y Métodos}

\section{Ubicación}

El estudio se realizó en el rebaño de alpacas Huacaya del Centro Experimental La Raya de la Universidad Nacional del Altiplano (UNA), ubicado entre 4100 y $5000 \mathrm{msnm}$ y al pie del Nudo Vilcanota de la Cordillera de los Andes. El territorio corresponde a la Puna Húmeda, y políticamente pertenece al distrito Santa Rosa, provincia Melgar, Puno (Perú).

El estudio empezó con la identificación de los animales en la esquila en noviembre de 2017 y concluyó con la toma de muestras en la siguiente esquila (octubre de 2018).

\section{Animales}

Se utilizaron 97 animales de la majada de alpacas Huacaya, siendo 41 machos y 56 hembras. Las edades de los machos fueron de 1,3 y 5 años y de las hembras de 1, 3, 5 y 7 años. La menor proporción de machos obedece a la presión selectiva establecida en el centro experimental para las campañas de empadre.

\section{Muestras y Mediciones}

En la esquila 2018 se extrajeron las muestras de fibras del costillar medio que correspondió a la parte media del vellón ( $3 \mathrm{~g} /$ animal). Las muestras fueron colocadas en sobres de papel rotulados.

Las mediciones fueron hechas sobre mechas crudas previamente corregidas en el equipo OFDA 2000 del Laboratorio de Fibras de la Universidad Nacional de Huancavelica. El equipo realiza mediciones de diámetro sobre fibras en secciones transversales de mecha de fibra cada $5 \mathrm{~mm}$ en toda su longitud, y con la secuencia de mediciones de cada mecha se obtiene el perfil de 
Cuadro 1. Promedios de diámetro medio de fibra, factor de confort, índice de curvatura y longitud de mecha de alpacas Huacaya según sexo y edad

\begin{tabular}{cccccc}
\hline Factor & $\mathrm{n}$ & $\begin{array}{c}\mathrm{DMF} \pm \mathrm{EE} \\
(\mu \mathrm{m})\end{array}$ & $\begin{array}{c}\mathrm{FC} \\
(\%)\end{array}$ & $\begin{array}{c}\mathrm{IC} \pm \mathrm{DE} \\
(\% \mathrm{~mm})\end{array}$ & $\begin{array}{c}\mathrm{LM} \pm \mathrm{DE} \\
(\mathrm{mm})\end{array}$ \\
\hline $\begin{array}{c}\text { Sexo } \\
\text { Macho }\end{array}$ & 41 & $20.90 \pm 0.39^{\mathrm{a}}$ & $95.98^{\mathrm{a}}$ & $37.57 \pm 7.09^{\mathrm{a}}$ & $95.97 \pm 16.52^{\mathrm{a}}$ \\
Hembra & 56 & $21.62 \pm 0.37^{\mathrm{a}}$ & $94.24^{\mathrm{a}}$ & $37.92 \pm 6.94^{\mathrm{a}}$ & $93.21 \pm 20.83^{\mathrm{a}}$ \\
Edad (años) & & & & & \\
1 & 39 & $19.48 \pm 0.25^{\mathrm{c}}$ & $98.15^{\mathrm{a}}$ & $34.82 \pm 5.52^{\mathrm{b}}$ & $102.18 \pm 12.92^{\mathrm{a}}$ \\
3 & 32 & $21.41 \pm 0.43^{\mathrm{b}}$ & $95.30^{\mathrm{a}}$ & $41.72 \pm 7.57^{\mathrm{a}}$ & $92.50 \pm 22.29^{\mathrm{a}}$ \\
5 & 18 & $23.57 \pm 0.47^{\mathrm{a}}$ & $91.09^{\mathrm{b}}$ & $38.09 \pm 6.45^{\mathrm{ba}}$ & $89.44 \pm 13.39^{\mathrm{ba}}$ \\
7 & 8 & $24.82 \pm 0.80^{\mathrm{a}}$ & $86.95^{\mathrm{c}}$ & $35.69 \pm 4.49^{\mathrm{b}}$ & $75.00 \pm 11.34^{\mathrm{b}}$ \\
\hline
\end{tabular}

$\mathrm{n}=$ Numero de observaciones, $\mathrm{DMF}=$ Diámetro medio de fibra, $\mathrm{FC}=$ Factor confort, $\mathrm{IC}=$ Índice de curvatura, $\mathrm{LM}=$ Longitud de mecha

diámetro de fibra (PDF). Por lo tanto, la cantidad de mediciones transversales guarda relación con la longitud de mecha.

Para la caracterización del Perfil de la Fibra (PDF) se determinaron tres puntos principales: i) Diámetro máximo (DMMax), diámetro mínimo inicial (DMMin1) entre el diámetro máximo y la punta de la mecha y el diámetro mínimo final y (DMMin2) entre el diámetro máximo y la base de la mecha. Además, se estimó, previo ajuste, la pendiente entre los respectivos DMMax y DMMin, dos tasas de cambio (TACA) del diámetro, la primera entre el DMMin1 y el DMMax (TACA1) y la segunda entre el DDMax y el DDMin2 (TACA2).

\section{Análisis Estadístico}

Los factores considerados fueron el sexo (macho y hembra) y la edad $(1,3,5$ y 7 años), todos de naturaleza fija. Se utilizó el modelo lineal aditivo, correspondiente a un Diseño Completamente Aleatorizado, conducido bajo un factorial de $2 \times 4$. El nivel de significación se realizó por comparación de medias Duncan a una probabilidad de $\mathrm{p}<0.05$. Para estimar la tasa de cambio, los valores del perfil de fibra fueron ajustados a un modelo de regresión no lineal de tipo cúbico; en tanto que, el PDF se ajustó a un modelo no lineal polinómico.

\section{Resultados y Discusión}

\section{Diámetro Medio de la Fibra}

El DMF no evidencia diferencias entre machos y hembras $(20.90 \pm 0.39$ y $21.62 \pm$ $0.37 \mu \mathrm{m})$. La variable edad mostró diferencias significativas $(\mathrm{p}<0.05)$, correspondiendo la mayor finura a las alpacas tuis menores $(19.48 \pm 0.25 \mu \mathrm{m})$, y el diámetro tiende a engrosar a partir de los 5 años de edad (Cuadro 1).

La ausencia de diferencias en el DMF por sexo concuerda con diversos autores (Flores, 1979); Siguayro, 2009; Ormachea et al., 2015; Arango, 2016; Quispe et al., 2016; 
Roque y Ormachea, 2018). Esto es corroborado por reportes llevados en otros espacios andinos (Wuliji et al., 2000; McGregor y Butler, 2004). No obstante, otros reportes nacionales (Álvarez, 1981; Montes et al., 2008; Vásquez et al., 2015; Ormachea et al., 2015; Machaca et al., 2017) o de animales llevados fuera del país (Aylan-Parker y McGregor, 2002; Lupton et al., 2006) indican diferencias de finura a favor del macho, lo cual reflejaría la mayor presión selectiva que se ejerce al elegir los reproductores para las campañas del empadre.

Con base a ello, y sin distinción de la ubicación de los estudios, se reporta que el DMF se incrementa significativamente con la edad del animal (Wuliji et al., 2000; Bustinza, 2001; McGregor y Butler, 2004; Ormachea et al., 2015; Quispe et al., 2016; Machaca et al., 2017; Roque y Ormachea, 2018). Por otro lado, los DMF hallados son menores respecto a los reportes de la misma región (Roque y Ormachea, 2018), con excepción de los estudios de Siguayro (2009) en Puno y de Arango (2016) en Cerro de Pasco y Machaca et al. (2018) en Apurímac. Más aún, las alpacas llevadas a otros continentes mostraron DMF elevados respecto al presente estudio (Lupton et al., 2006; McGregor, 2006). En consecuencia, la expresión del DMF está influenciado por la edad del animal y por factores ambientales (ubicación agroecológica, nivel altitudinal).

\section{Factor de Confort}

Los promedios del FC fueron similares para machos (95.98\%) y hembras (94.24\%), tal y como lo indican Roque y Ormachea (2018), aunque otros autores reportaron diferencias entre sexos (Ormachea et al., 2015; Vásquez et al., 2015; Machaca et al., 2017). De otra parte, se coincide con trabajos reportados sobre diferencias significativas respecto a la edad (Ormachea et al., 2015; Vásquez et al., 2015; Machaca et al., 2017; Roque y Ormachea, 2018). Es posible señalar que, hasta la edad de tres años, las alpacas exhiben altos índices de confort (98.15 y
95.30\%), para decaer conforme avanza la edad del animal $(\mathrm{p}<0.05)$ (Bustinza et al., 1985; Quispe, 2018).

Estas diferencias se acentúan en alpacas en países donde han sido introducidas (Lupton et al., 2006). En consecuencia, se infiere que a medida que las alpacas completan su ciclo productivo producen fibras gruesas -cada vez más rígidas o inflexiblesque son responsables de la sensación de irritación o picor sobre la piel humana. En tal perspectiva, el FC indica el umbral de comodidad de la fibra, o sea es el porcentaje de fibras con valor inferior a $30 \mu \mathrm{m}$ (Botha y Hunter, 2010).

El DMF y el FC muestran comportamiento particular; pues en los animales adultos la proporción de fibras mayores de $30 \mu \mathrm{m}$ excede el 5\%; en virtud de ello, solo la fibra proveniente de alpacas menores de tres años cumpliría las exigencias de comodidad de los consumidores.

\section{Índice de Curvatura}

El IC de las fibras de alpaca fue similar entre sexos (Machos 37.57 \pm 7.09 : Hembra: $37.92 \pm 6.94 \% \mathrm{~mm}$ ), pero significativamente diferente entre edades $(p<0.05)$, variando entre $34.82 \pm 5.52$ y $41.72 \pm 7.58 \% \mathrm{~mm}$. No obstante, a diferencia del DMF o el FC, el IC de las edades extremas del estudio (1 y 7 años) fueron similares (Cuadro 1), lo que evidencia que el IC no muestra una tendencia regular como si se observa en el DMF y el FC.

Al respecto, en alpacas Huacaya de 1 año, Marín (2007) reporta 47.14 y $47.22 \% \mathrm{~mm}$ para hembras y machos, respectivamente, mientras que Siguayro (2009) en Lampa, Puno, reporta valores de 54.70 y $54.01 \% \mathrm{~mm}$ para machos y hembras, respectivamente. Valores de IC similares entre sexos fueron también reportados por Ormachea et al. (2015), Vásquez et al. (2015) y Roque y Ormachea (2018). En cambio, Machaca et al. (2017) hallaron IC favorables para alpacas macho 
Cuadro 2. Valores de los diámetros medio mínimo inicial $\left(\operatorname{Min}_{1}\right)$ y final $\left(\operatorname{Min}_{2}\right)$, diámetro medio máximo (DMMax) y tasa de cambio (TACA), $\mu \mathrm{m} / \mathrm{mm}$ ) de la fibra de alpacas Huacaya según sexo y edad (promedio \pm EE)

\begin{tabular}{cccccc}
\hline Factor & DMMin $_{1}$ & DMMax & DMMin $_{2}$ & TACA $_{1}$ & TACA $_{2}$ \\
\hline Sexo & & & & & \\
Macho (n) & 39 & 39 & 39 & 39 & 39 \\
& $20.22 \pm 0.31^{\mathrm{a}}$ & $23.39 \pm 0.41^{\mathrm{a}}$ & $19.69 \pm 0.43^{\mathrm{a}}$ & $0.0482^{\mathrm{b}}$ & $-0.0746^{\mathrm{a}}$ \\
Hembra (n) & 53 & 53 & 53 & 53 & 53 \\
& $20.24 \pm 0.26^{\mathrm{a}}$ & $24.16 \pm 0.45^{\mathrm{a}}$ & $20.44 \pm 0.35^{\mathrm{a}}$ & $0.0725^{\mathrm{a}}$ & $-0.0795^{\mathrm{a}}$ \\
Edad (años) & & & & & \\
1 (n) & 35 & 35 & 35 & 38 & 38 \\
& $19.117 \pm 0.18^{\mathrm{b}}$ & $21.63 \pm 0.34^{\mathrm{c}}$ & $18.07 \pm 0.252^{\mathrm{c}}$ & $0.04817^{\mathrm{b}}$ & $-0.0563^{\mathrm{a}}$ \\
3 (n) & 30 & 30 & 30 & 28 & 28 \\
5 (n) & $20.03 \pm 0.339^{\mathrm{b}}$ & $24.48 \pm 0.399^{\mathrm{b}}$ & $20.367 \pm 0.34^{\mathrm{b}}$ & $0.072509^{\mathrm{a}}$ & $-0.09606^{\mathrm{b}}$ \\
7 (n) & 19 & 19 & 19 & 19 & 19 \\
& $21.95 \pm 0.376^{\mathrm{a}}$ & $25.64 \pm 0.523^{\mathrm{ba}}$ & $22.426 \pm 0.36^{\mathrm{a}}$ & $0.06756^{\mathrm{b}}$ & $-0.0669^{\mathrm{ba}}$ \\
& 8 & 8 & 8 & 8 & 8 \\
\hline
\end{tabular}

TACA1: entre DMMin 1 y DMMax; TACA2: entre DDMax y DDMin ${ }^{2}$

en la región Apurímac. En Australia, Nueva Zelanda y EEUU se reportan valores bajos de IC que oscilan de 28.0 a $32.5^{\circ} / \mathrm{mm}$ (Liu et al., 2004; Wang et al., 2004; Lupton et al., 2006; McGregor, 2006).

En términos generales y según los trabajos revisados, las fibras de las alpacas corresponden a la categoría bajo índice de curvatura, excepto las alpacas del estudio de Machaca et al. (2017) que se clasificarían entre bajo y mediano índice de curvatura. Allí reside la utilidad del IC, pues permite predecir la fuerza de cohesión, la capacidad de hilado y rendimiento de la fibra (Edmonds, 1997). Más aún, si se toma en cuenta que el IC está asociado al rizo de la mecha (frecuencia de ondulación), por su particular influencia sobre la cohesión y el rendimiento en el hilado (Botha y Hunter, 2010).

\section{Longitud de Fibra}

La LF fue similar entre sexos (Macho: $95.97 \pm 16.52$; Hembra: $93.21 \pm 20.83 \mathrm{~mm}$ ), mientras que fue significativamente diferente entre edades ( $<<0.05$; Cuadro 1 ), indicando que hasta los primeros cinco años muestran el mayor crecimiento longitudinal de la fibra, para luego disminuir gradualmente $(p<0.05)$; resultados similares a los encontrados por Roque y Ormachea (2018).

La importancia de la LF radica en su vinculación con el rendimiento del procesamiento de la fibra; pues las fibras largas son más deseables comercialmente, ya que tienden a ser más fáciles de hilar, dan menos paros y, en última instancia, pueden formar hilos más fuertes y uniformes en comparación de las fibras más cortas (Holman y Malau-Aduli, 2012). 


\section{Perfil del Diámetro de Fibra (PDF)}

Los principales parámetros utilizados para caracterizar el PDF de alpacas Huacaya se presenta en el Cuadro 2 y en las figuras 1 y 2, por factores sexo y edad.

\section{Diámetros mínimos y máximos}

Se ha diferenciado dos diámetros mínimos $\left(\mathrm{DMMin}_{1}\right.$ y $\left.\mathrm{DMMin}_{2}\right)$ y un diámetro máximo (DMMax). Los primeros se encuentran a la izquierda y derecha del DMMax. Para el factor sexo, los DMMin no muestran diferencias y ambas oscilan alrededor de 20 $\mu \mathrm{m}$, en tanto que el DMMax mostró ligeras diferencias para machos y hembras (Cuadro 2). Para estos parámetros, las alpacas mayores de 5 años mostraron los valores más altos respecto a las de menor edad $(\mathrm{p}<0.05)$.

La magnitud de las diferencias del DMMin respecto al DMMax varían en función a la edad. Es evidente que los tuis de 1 año exhiben la mayor finura.

Aquel comportamiento, con base al DMMax permite configurar una curva compuesta de tres segmentos: uno ascendente y pronunciado, otra a manera de una meseta sostenida y convexa y el tercero que desciende lentamente, lo cual revelaría el patrón de comportamiento del DMF durante la campaña ganadera. En sí, aquel coincide con el ciclo fenológico de las especies de la pradera andina condicionada por la estacionalidad de la precipitación anual (Quispe et al., 2016; Quispe, 2018). En tanto, los valores de los DMMin y DMMax se encuentran dentro del rango reportado para alpacas de la sierra central del país, los que fueron agrupados con base a la frecuencia de esquila (Poma y Ventura, 2009).

\section{Tasas de Cambio}

Con base a la configuración de una curva y los parámetros del Cuadro 2, se dispone de dos tasas de cambio (TACA y TACA $_{2}$ ) que muestran valores positivos y negativos, res- pectivamente, con las atingencias siguientes: la TACA $A_{1}$, antes del DMMax mostró diferencias a favor de la hembra $(\mathrm{p}<0.05)$, y las $\mathrm{TACA}_{2}$ fueron relativamente similares. En sí, la tasa de cambio es el cambio de diámetro de fibra expresada en micras por milímetro $(\mu \mathrm{m} / \mathrm{mm})$ que ocurre durante el crecimiento en un periodo de tiempo a lo largo de la mecha (Sacchero y Mueller, 2007; Sacchero et al., 2010). Sin embargo, para el factor edad, las TACA de alpacas de 7 años es proporcionalmente el doble respecto a las otras edades; lo cual puede relacionarse al periodo del estudio (noviembre-octubre), que a diferencia de Poma y Ventura (2009), las alpacas de más edad muestran mayor sensibilidad a los cambios estacionales; en particular, a la disponibilidad de nutrientes en la pradera andina, la cual se acrecienta al final del periodo lluvioso (Flórez y Malpartida, 1987; Bustinza, 2001; Quispe et al., 2016; Quispe, 2018).

La comparación de las $\mathrm{TACA}_{1}$ y $\mathrm{TACA}_{2}$ hallados en alpacas tuis de 1 año son similares a lo determinado en alpacas de la misma edad, a nivel de la sierra central (Lacchocc, Huancavelica); empero los animales con mayor frecuencia de esquila exhiben valores de cambio más altas, sea en el lado ascendente o descendente del PDF (Poma y Ventura, 2009). Ello corrobora que las alpacas tuis de 1 año son más estables en su respuesta, variable DMF, a los cambios nutricionales que obran a su alrededor; en tanto que la respuesta de los animales de mayor edad es más sensible ante dichas variaciones.

De la misma manera, las TACA 1 y $\mathrm{TACA}_{2}$ muestran valores positivos y negativos, respectivamente; con la atingencia que ambas tasas mostraron diferencias significativas, aunque pequeñas, en función a la edad $(p<0.05)$. Se reitera, con base a la consideración del periodo de estudio (noviembre-octubre), que no hubo clara diferenciación de los cambios estacionales (Flórez y Bryant, 1985; Bustinza, 2001; Quispe, 2018). En ovejas Merino del noroeste de la Patagonia Argenti- 


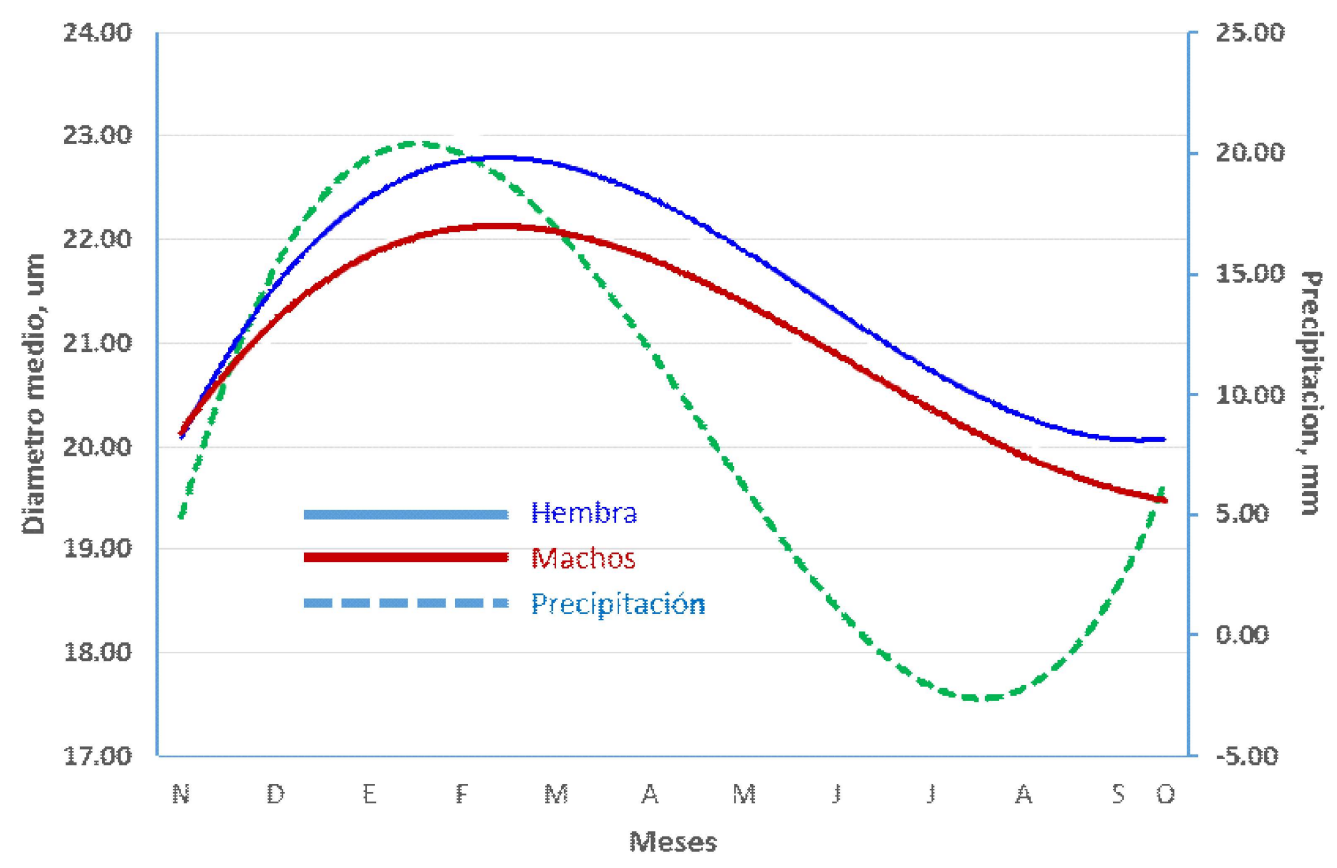

Figura 1. Perfil del diámetro de fibra de alpacas Huacaya por factor sexo. Curva de precipitación adaptado de Quispe (2018)

na, las TACA fueron influenciadas no solo por las condiciones ambientales del área de estudio, sino también por la línea genética, donde las TACA reportados antes del DMMax fueron más altos respecto a las TACA después de ella (Sacchero et al., 2010).

\section{Perfil del diámetro de fibra}

Con base a los parámetros descritos en los rubros anteriores y las mediciones del DMF a lo largo de la fibra se construyó el PDF de las alpacas en estudio por los factores sexo (Figura 1) y edad (Figura 2). Las figuras reflejan el patrón de variabilidad del diámetro de fibra ocurrido durante una campaña alpaquera (noviembre-diciembre).

Para el factor sexo, el PDF asciende rápidamente para luego descender gradualmente hasta alcanzar su valor mínimo próximo a la siguiente esquila. El PDF descrito guarda relación con el perfil de la precipitación anual ocurrida en el Altiplano peruano, que se caracteriza por una época lluviosa corta (diciembre-marzo) y otra larga en la época de estío (abril-noviembre) (Quispe et al., 2016; Quispe, 2018). Los modelos polinómicos pertinentes para machos $\mathrm{y}$ hembras fueron: $y=0.0021 \times 3-0.0942 \times 2+$ $1.0767 \mathrm{x}+19.105\left(\mathrm{R}^{2}=0.9716\right)$ y $y=$ $0.0015 \times 3-0.0685 \times 2+0.7919 x+19.417\left(R^{2}\right.$ $=0.9726)$, los que son propios para las características de naturaleza biológica. Esta estacionalidad guarda relación directa con la producción de pastos (disponibilidad de nutrientes). Obviamente, también deben considerarse las prácticas de manejo de pastos establecido en el Centro Experimental. Bajo esa consideración, el PDF, poco después del inicio de la época lluviosa, evidencia el engrosamiento del DMF, cuyo efecto se prolonga hasta el $50 \%$ de la longitud de la fibra, para luego tender a afinarse. 


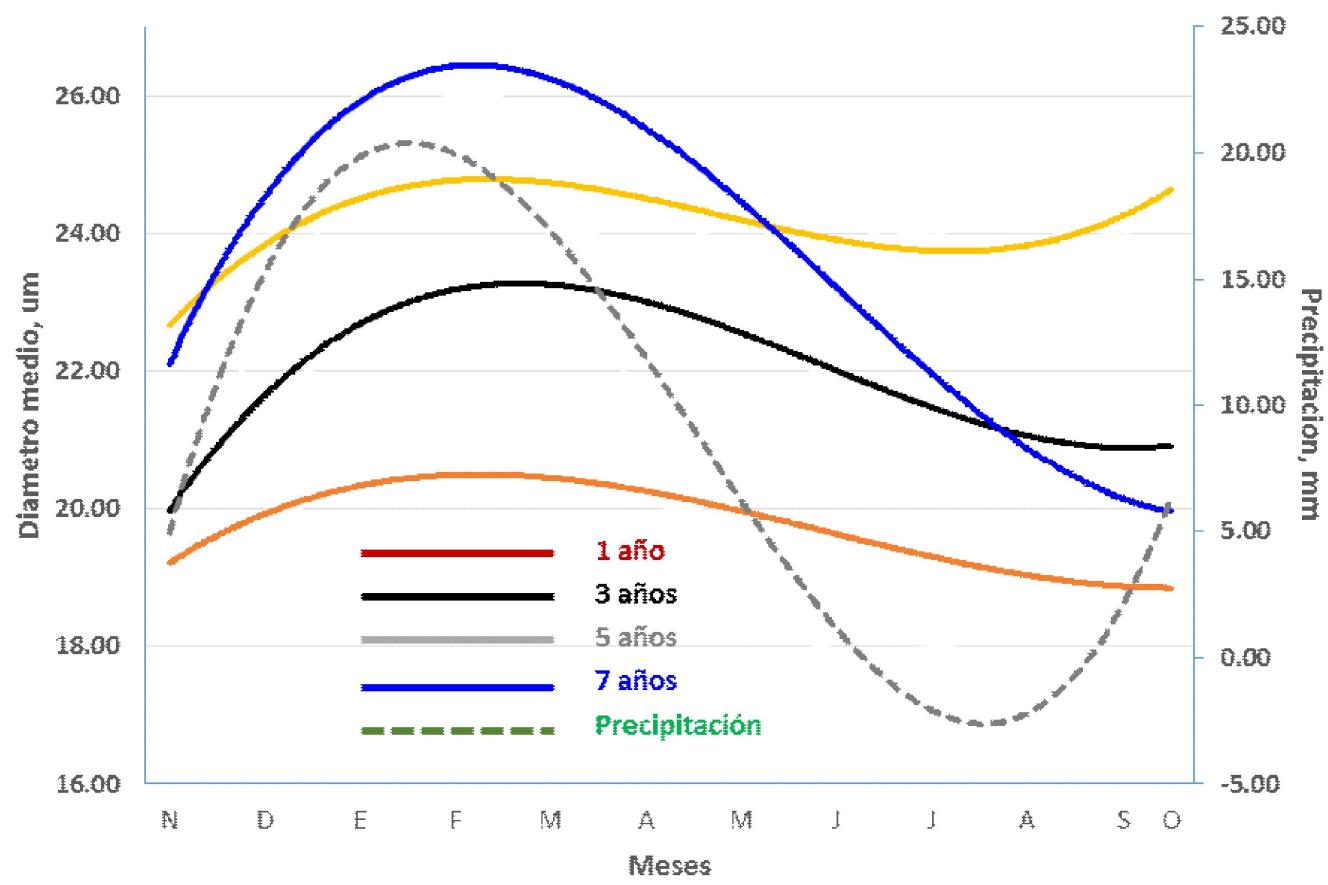

Figura 2. Perfil del diámetro de fibra de alpacas Huacaya por factor edad. Curva de precipitación adaptado de Quispe (2018)

A nivel general, lo delineado en la Figura 1 evidencia que la esquila se da próximo al DMMin, lo cual constituye una buena práctica de manejo; pues no disturba el uso potencial de la fibra en la industria textil.

La Figura 2, para el factor edad, a diferencia de lo observado para el factor sexo, el perfil de las alpacas hasta prácticamente los 5 años es menos sensible a la variabilidad de las condiciones ambientales (disponibilidad de nutrientes). Las alpacas de 7 años, por otro lado, son más sensibles, pues a pesar de tener el mayor valor del DMF, tienden a afinarse más rápidamente. Ello, en sí sería el comúnmente denominado finura de hambre, probablemente atribuibles a la menor actividad metabólica propia de los animales adultos (Bustinza, 2001; Quispe et al., 2016; Quispe, 2018).
Los modelos pertinentes por edades fueron: $\mathrm{Y}_{(1)}=0.0011 \mathrm{x}^{3}-0.0478 \mathrm{x}^{2}+0.5346 \mathrm{x}+$ $18.714\left(\mathrm{R}^{2}=0.9362\right)$ para alpacas de un año; $\mathrm{Y}_{(3)}=0.0023 \mathrm{x}^{3}-0.1017 \mathrm{x}^{2}+1.219 \mathrm{x}+18.8478$ $\left(\mathrm{R}^{2}=0.9401\right)$ para 3 años; $\mathrm{Y}_{(5)}=0.0022 \mathrm{x}^{3}$ $-0.0843 \mathrm{x}^{2}+0.8996 \mathrm{x}+21.839\left(\mathrm{R}^{2}=0.8559\right)$ $\mathrm{y} \mathrm{Y}_{(7)}=0.0036 \mathrm{x}^{3}-0.1634 \mathrm{x}^{2}+1.8227 \mathrm{x}+$ $20.436\left(\mathrm{R}^{2}=0.9622\right)$ para 7 años. El comportamiento no lineal del PDF de la fibra de alpacas por edad obedece a su naturaleza genética y factores ambientales que rodean a la crianza (Quispe et al., 2016; Quispe, 2018).

\section{Conclusiones}

- Los promedios de diámetro, factor de confort, índice de curvatura y longitud de mecha de la fibra de las alpacas 
Huacaya del Centro Experimental La Raya fueron similares entre sexos. No obstante, presentan una relación directa con el avance de la edad para el diámetro medio de fibra y longitud de mecha, y una relación inversa para el factor de confort.

- Los parámetros que definen el perfil de la fibra por sexo fueron similares, mientras que guardan relación con la edad de la alpaca.

- En conjunto, el perfil de la fibra tiene relación con la estacionalidad de la producción de pastos en la pradera andina.

\section{Literatura Citada}

1. Álvarez J. 1981. Dimensiones físicas de la fibra de alpaca en la CAP Huaycho Ltda. $\mathrm{N}^{\circ} 44$. Tesis de Médico Veterinario Zootecnista. Puno, Perú: Univ. Nacional del Altiplano. $76 \mathrm{p}$.

2. Arango S. 2016. Variaciones del factor de confort en vellones de alpaca Huacaya con relación al sexo y edad. Tesis de Ingeniero Zootecnista. Lima, Perú: Univ. Nacional Agraria La Molina. 56 p.

3. Antonini M, Vinella S. 1997. Fine fibre production from Argentine camelids. A development perspective. European Fine Fibre Network, Occasional Publication 6: 31-41.

4. Aylan-Parker J, McGregor BA. 2002. Optimizing sampling techniques and estimating sampling variance of fleece quality attributes in alpacas. Small Ruminant Res 44: 53-54. doi: 10.1016/ S0921-4488(02)00038-X

5. Bustinza AV, Sapana R, Medina G. 1985. Crecimiento de la fibra de alpaca durante el año. En: Memorias del Proyecto Piel de Alpaca. Puno, Perú: Univ. Nacional del Altiplano.

6. Bustinza V. 2001. La alpaca: conocimiento del potencial andino. Libro 1. Puno, Perú: Univ. Nacional del Altiplano. $495 \mathrm{p}$.
7. Cardellino RC, Mueller JP. 2008. Fibre production and sheep breeding in south America. Proc Assoc Advmt Anim Breed Genet 18: 366-373.

8. Edmonds ER. 1997. Measurement of fibre curvature: a review of work to date. Wool Tech Sheep Bree 45: 227-234.

9. Flores H. 1979. Diámetro y longitud de mecha en alpacas Wacaya y Sury machos y hembras de 1 a 6 años de edad del CE La Raya. Tesis de Médico Veterinario Zootecnista. Puno, Perú: Univ. Nacional del Altiplano. $81 \mathrm{p}$.

10. Flórez E, Malpartida E. 1987. Manejo de praderas nativas y pasturas en la región altoandina del Perú. Lima, Perú: Fondo Editorial del Libro de Banco Agrario. $651 \mathrm{p}$.

11. Flórez A, Bryant F. 1985. Fenología y contenido de nutrientes de gramíneas claves en los Andes de Perú. En: Investigación sobre pastos y forrajes de Texas Tech University en el Perú. Vol II. Red Rumen - INIA Perú. Lubbock, USA. p 13-17.

12. Liu X, Wang L, Wang X. 2004. Evaluating the softness of animal fibers. Text Res J 74: 535-538. doi: 10.1177/ 004051750407400612

13. Lupton J, Mccoll A, Stobart RH. 2006. Fiber characteristics of the Huacaya alpaca. Small Ruminant Res 64: 211-224. doi: 10.1016/j.smallrumres.2005.04.023

14. Machaca V, Bustinza V, Corredor F, Paucara V, Quispe E, Machaca R. 2017. Características de la fibra de alpaca Huacaya de Cotaruse, Apurímac, Perú. Rev Inv Vet Perú 28: 843-851. doi: 10.15381/rivep.v28i4.13889

15. Marín E. 2007. Efecto del sexo sobre las características tecnológicas y productivas en alpacas tuis para su uso en la industria textil. Tesis de Maestría. Lima, Perú: Univ. Nacional Agraria La Molina. $113 \mathrm{p}$.

16. McGregor BA, Butler KL. 2004. Sources of variation in fibre diameter attributes of Australian alpacas and implications for fleece evaluation and animal selection. Aust J Agr Res 55: 433442. doi: 10.1071/AR03073 
17. McGregor BA. 2006. Production, attributes and relative value of alpaca fleeces in southern Australia and implications for industry development. Small Ruminant Res 61: 93-111. doi: 10.1016/j.smallrumres.2005.07.001

18. MINCETUR. 2018. Perfil de prendas de alpaca del Mercado de Estados Unidos. $102 \mathrm{p}$.

19. Montes M, Quicaño I, Quispe R, Quispe EC, Alfonso L. 2008. Quality characteristics of Huacaya Alpaca fibre produced in Peruavian Andean plateau region of Huancavelica. Span J Agric Res 6: 33-38. doi: 10.5424/sjar/2008061-5258

20. Ormachea E, Calsín B, Olarte $U$. 2015. Características textiles de la fibra en alpacas Huacaya del distrito de Corani Carabaya, Puno. Rev Investig Altoandin 17: 215-220. doi: 10.18271/ RIA.2015.115.

21. Poma AG, Ventura CE. 2009. Caracterización del perfil de diámetro de fibra de alpaca Huacaya del color blanco. Tesis de Ingeniero Zootecnista. Huancavelica, Perú: Univ. Nacional de Huancavelica. $99 \mathrm{p}$.

22. Quispe JE, Apaza E, Quispe DM, Morocco N. 2016. De vuelta a la alpaca: la producción primaria en una perspectiva empresarial y competitiva. Puno, Perú: Univ. Nacional del Altiplano. $450 \mathrm{p}$.

23. Quispe JE. 2018. Efectos biológicos y ambientales sobre las características de producción de carne y fibra de alpacas del CIP Quimsachata - INIA Puno Perú. Tesis Doctoral. Puno, Perú: Univ. Nacional del Altiplano. $210 \mathrm{p}$.

24. Roque L, Ormachea E. 2018. Características productivas y textiles de la fibra en alpacas Huacaya de Puno, Perú. Rev Inv Vet Perú 29: 1325-1334. doi: 10.15381/rivep.v19i4.14117

25. Sacchero DM, Mueller JP. 2007. Diferencias en el perfil de diámetro de fi- bras, largo de mecha y resistencia a la tracción de la lana, en ovejas de una majada Merino seleccionada y otra no seleccionada. Rev Invest Agropec 36: 49-61.

26. Sachero D, Willems P, Mueller JP. 2010. Perfiles de diámetro de fibras en lanas preparto de ovejas merino. 1. Estudio comparativo de líneas genéticas. Rev Arg Prod Anim 30: 31-42.

27. Siguayro R. 2009. Comparación de las características físicas de las fibras de la llama Ch'aku (Lama glama) y la alpaca Huacaya (Lama pacos) del Centro Experimental Quimsachata del INIA Puno. Tesis de Maestría. Lima, Perú: Univ. Nacional Agraria La Molina. $88 \mathrm{p}$.

28. Vásquez R, Gómez-Quispe O, Quispe E. 2015. Características tecnológicas de la fibra blanca de alpaca Huacaya en la zona altoandina de Apurímac. Rev Inv Vet Perú 26: 213-222. doi: 10.15381/ rivep.v26i2.11020

29. Wang L, Liu X, Wang X. 2004. Changes in fibre curvature during the processing of wool and alpaca fibres and their blends. In: Proc Textile Institute $83^{\text {rd }}$ World Conference. England: The Textile Institute.

30. Wang X, Wang L, Liu X. 2003. The quality and processing performance of alpaca fibres: Australian alpaca fibre industry and the fibre properties. Rural Industries Research and Development Corporation. RIRDC Publication No 03/ 128. Project No. UD-2A.

31. Wuliji T, Davis GH, Dodds GK, Turner PR, Andrews RN, Bruce GD. 2000. Production performance, repeatability and heritability estimates for live weight, fleece weight and fiber characteristics of alpacas in New Zealand. Small Ruminant Res 37: 189-201. doi: 10.1016/ S0921-4488(00)00127-9 